\title{
Site Specific Cryo-FIB Preparations Aimed at in situ Cryo-Electron Tomography
}

Julia Mahamid $^{1}$, Jan Arnold ${ }^{1}$ and Jürgen M. Plitzko ${ }^{1}$

${ }^{1}$ Department of Molecular Structural Biology, Max Planck Institute of Biochemistry, Martinsried 82152, Germany

The function of many macromolecular assemblies is so deeply rooted in their cellular context that it is impossible to isolate them without compromising their structural integrity. Hence, it is highly desirable to study them in situ - within an unperturbed cell in which they perform their function. Recent advances in cryo-focused ion beam (FIB) micromachining bring 'large' mammalian cells into the range of electron transparency $(100-300 \mathrm{~nm})$ [1], and cryo-electron tomography (ET) on these preparations now provides marvelous molecular views in cells [2].

Interpretation of these views within the context of specific cellular functions is the next goal [3]; the volume represented within a typical FIB lamella constitutes a small fraction of the biological specimen. Thus, retaining low-abundance and dynamic subcellular structures, arrested by vitrification, within such limited volumes requires precise targeting of the FIB milling process. To answer these needs, we describe the incorporation of confocal light microscopy (LM) at cryogenic temperatures into existing workflows for cellular sample preparation by cryo-FIB (Figure 1). By introducing microspheres as fiducial markers that are distinguished both in LM and the scanning electron microscope (SEM)/FIB, we compute a 3D 'rigid-body' coordinate transformation. The correlation accuracy attained with the aid of fiducial markers surpasses the physical resolution of the LM and allows targeting within the range of tens of nanometers.

A software encompassing the necessary functions to calculate $3 \mathrm{D}$ correlation is under open source development ([4] and Figure 1: II). This includes data preprocessing tools of LM confocal stacks and 3D coordinate extraction functions that determine the location of markers in the LM volume semiautomatically. Appropriate sample positions, judged by the number and distribution of markers, are selected prior to the SEM/FIB session. Markers in the SEM and FIB images are then selected manually by the user, which introduces errors and a possible propagation of uncertainty. Thus, after an initial correlation, visual guides indicate deviation of the selected markers from their respective calculated positions. This helps the user to identify manual selection bias, eliminate markers with large deviations and allows for adjustments, leading to minimization of those errors. The process is conducted while operating the SEM/FIB and allows for on-line 3D correlation and immediate site-specific cryo-FIB preparation (Figure 1: III).

The correlative approach was employed to navigate FIB milling and to capture specific structures, lipid droplets as an example, in vitrified cellular samples [3]. The correlation approach can then be applied to navigate the acquisition of cryo-ET data within the FIB-lamella at specific locations, unambiguously identified by fluorescence microscopy (Figure 1: IV).

We will discuss approaches to improve the localization specificity and precision in $3 \mathrm{D}$, the preparation reproducibility and yield, all together key factors in advancing in situ imaging for structural biology. 

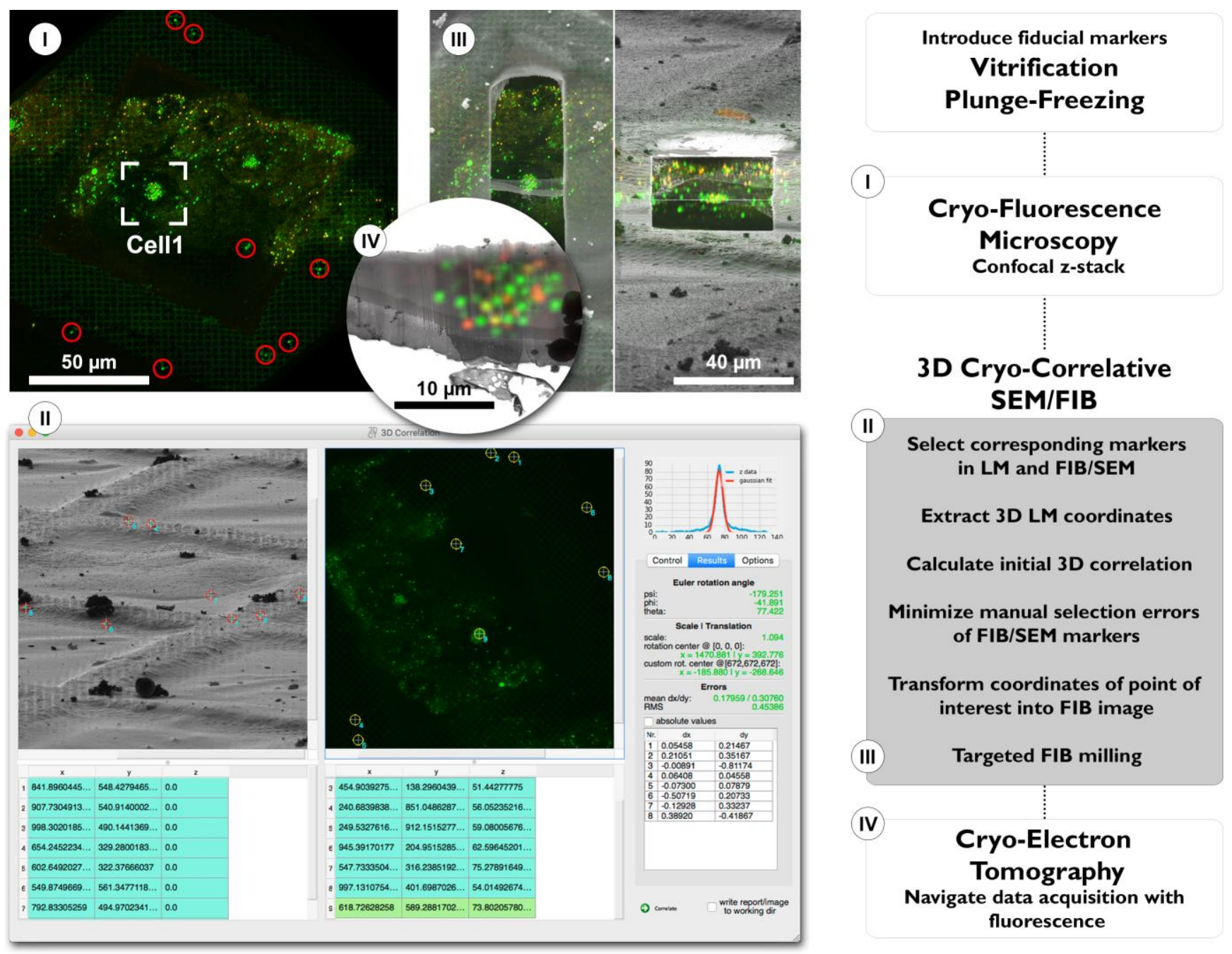

Figure 1. 3D correlation applied to HeLa cell culture for targeting fluorescently labeled lipid droplets. Right: A diagram of the 3D correlative workflow. Left: I. Maximum intensity projection of a confocal volume. Green: general neutral lipid dye and magnetic beads used as fiducials (encircled; levels were adjusted separately inside encircled areas for better visualization of the fiducials). Red: neutral fatty acids dye. II. Graphical user interface of the 3D correlation software. A thin lamella is produced following correlation by FIB milling. III. SEM and FIB images overlaid with LM data after applying transformation. IV. Cryo-Transmission electron microscopy montage of FIB-lamella overlaid with a computer-generated slice through the confocal volume. Lipid droplets stained in green or red are distinguishable within the lamella.

\section{References:}

[1] M Schaffer et al., Journal of Structural Biology 2016, 197(2): 73-82.

[2] J Mahamid et al., Science 2016, 351(6276): 969-672.

[3] J Arnold et al., Biophysical Journal 2016, 110(4): 860-869.

[4] https://3dct.semper.space/

[5] JM acknowledges funding from EMBO and HFSP postdoctoral fellowships. 